Pascal M, Homer CSE. Models of postnatal care for low-income countries: A review of the literature. International Journal of Childbirth, 6(2): 104-132.

\title{
MODELS OF POSTNATAL CARE FOR LOW-INCOME COUNTRIES: A REVIEW OF THE LITERATURE
}

\section{ABSTRACT}

PURPOSE: This review aims to identify the key features of effective models of postnatal care and to determine which elements may be appropriate for low income countries.

StUdy DeSIGN: A narrative synthesis of English-language, peer-reviewed articles from 2004 to 2014 was undertaken. Five online library databases were searched. Inclusion/exclusion criterion and a quality appraisal were applied.

MAJOR FINDINGS: 22 studies were included in the review; four were from low income countries. Postnatal care was provided both in hospital and community settings and midwives were identified as the predominant and the most appropriate professionals to provide postnatal care in every location. A midwife-led model could provide high-quality, woman-centred, cost-effective care and ensure continuity of care. Inter-professional collaborative models were seen as useful especially in contexts of workforce shortage, though required improvement in the provision of support and coordination between providers to ensure appropriate skill-mix. Midwifery-led and inter-professional collaborative models could be adapted and implemented in low-income countries to provide quality postnatal care.

MAIN CONCLUSION: Low-income countries could develop a midwifery-led model together with interprofessional models for the provision of quality postnatal care. Education and practice will need to be addressed as well as promotion to the population.

KEY WORDS: maternity; midwifery; postnatal care; model of care; postnatal period; midwife-led. 


\section{INTRODUCTION}

In 2013, the World Health Organization (WHO) reported that 289,000 women died because of pregnancy and childbirth complications (World Health Organization 2014) and two million children died during their first week of life (Save the Children, 2013). Almost all these deaths, $99 \%$ in both women and children, occurred in low income countries (Save the Children 2013). Mothers mostly die of haemorrhage, infection, unsafe abortion, eclampsia and obstructed labour (World Health Organization, 2014) while newborns die from complications of preterm birth, intra-partum related events, sepsis and meningitis, pneumonia and congenital abnormalities (World Health Organization (WHO), 2014). However, the lack of access to basic health care services before, during and after birth is greatly responsible for the majority of deaths. For many mothers and newborns around the world, essential health care is often unavailable, inaccessible, unaffordable or of poor quality (The State of the World's Midwifery, 2014; World Health Organization, 2014; World Health Organization (WHO), 2014).

While numerous actions have been undertaken to increase the rate of skilled care provided during pregnancy and birth and to improve the quality of care for mothers and babies (The State of the World's Midwifery, 2014; World Health Organization (WHO), 2014), the postnatal period has often been neglected. This is a critical period in improving the health and wellbeing of mothers and newborns and needs urgent consideration. The WHO has made specific recommendations about the content of postnatal care, in particular, the provision of care in resource-limited settings and in low and middle-income countries. These recommendations describe the timing, number of contacts and content of the postnatal care to provide (World Health Organization, 2013b). Furthermore, the WHO's Every Newborn Action Plan (World Health Organization (WHO) 2014) provides a series of global actions and priorities to improve newborn health and reduce the incidence of stillbirth by 2035. These include high quality postnatal care, especially in the first hours and days after birth - the most critical time. Postnatal care for the normal newborn includes early initiation of breastfeeding, prevention of hypothermia, clean postnatal care practices and appropriate cord care, and the detection of problems requiring additional care. Postnatal care is delivered in various settings, by different providers, at different times. In order to provide this care, different models of postnatal care have been established across the world, designed to address specific constraints and contexts.

A trained health workforce and effective models of care are critical to being able to provide effective postnatal care, especially in low-income countries. The State of the World's Midwifery (SoWMy) report developed Midwifery 2030, which is a vision that can be used to guide Maternal, Newborn and Child Health (MNCH) services development (The State of the World's Midwifery, 2014). This vision highlighted the importance of quality postnatal care to improve the health of mothers and newborns in low income countries. However, it is still unknown which models or approaches to providing postnatal care would be most effective and sustainable in low-income countries. This paper is the synthesis of a literature review we conducted to identify the features of the different models of postnatal care that are currently in place in low and high-income countries. The review aims are to:

- identify the key features of effective models of postnatal care.

- determine which models may be appropriate for implementation in low income countries. 


\section{MATERIAL AND METHODS}

A narrative synthesis methodology was used. This approach has been used in a number of similar literature reviews where there is limited understanding about a single topic (Dawson, Buchan, Duffield, Homer, \& Wijewardena, 2013; Shahheidari \& Homer, 2012). In this review, qualitative and quantitative literature were reviewed and synthesised to address the two aims of the study.

\section{SEARCH PROTOCOL}

A systematic review of English language, peer-reviewed literature published from 2004-2014 was undertaken. We searched through five main online bibliographic databases for health, that is, EBSCO, CINAHL, Ovid MEDLINE, and Cochrane Database of Systematic Reviews, as well as Google Scholar.

The key words used were 'maternity', 'midwifery', 'postnatal care', 'model of care', 'postnatal period', and 'midwife-led'.

The reference lists of included articles were checked to determine other articles for inclusion. Studies written in English that evaluated models of postpartum/postnatal care and their impact on mothers and babies, staff and families were included. We decided to select only primary research papers and thus excluded literature reviews and systematic reviews.

We identified 45 studies that met the inclusion criteria and retrieved all the abstracts. The retrieved records were screened to select the studies focusing on models of postnatal care or models of maternity care that included postnatal care.

\section{QUALITY APPRAISAL}

The full papers were appraised to ascertain the alignment of the research aim and methodology with our aim, as well as to evaluate the recruitment, settings, data analysis, ethical considerations, findings and contribution to knowledge according to the CASP Systematic Review Checklist method (CASP UK, 2014). We excluded 20 studies as they did not meet with the requirements of our aims. Amongst the 25 remaining studies, we selected only primary research and excluded three literature reviews. This review, therefore, includes a total of 22 studies (Figure 1), 12 descriptive studies: six qualitative, six quantitative, and 11 analytic studies including three randomised studies, three cohort study designs and four cross sectional analytic designs.

\section{SYNTHESIS OF THE FINDINGS}

A narrative synthesis approach was conducted on the 22 final papers. We used tables to organise the information extracted from studies. We classified the content by theme.

\section{FINDINGS}

Nineteen of the 22 articles (Table 1) focused on postnatal care in high-income countries including Australia ( $n=13)$, the United Kingdom (UK) $(n=1)$, Sweden $(n=1)$, Finland $(n=1)$, Taiwan $(n=1)$ and Canada $(n=1)$. Five articles focussed on postnatal care in low and middle-income countries, those being, Bangladesh ( $n=2)$, India $(n=1)$, and Syria $(n=1)$. 
The majority of studies focused on analysis of models of postnatal care provided in a specific location such as maternity unit $(n=11)$ or in the community $(n=7)$. The second focus was on the type of providers involved in the models of care including non-medical providers: midwifery-led model $(n=6)$, community health workers model $(n=1)$, collaborative models $(n=2)$, and medical providers $(n=2)$.

\section{LOCATION OF CARE - WHERE DOES POSTNATAL CARE OCCUR?}

Two locations were identified as the main settings for the provision of postnatal care; they were hospital and the community. Community settings were either home or community facility.

\section{What happens at hospital?}

All the studies that examined hospital-based care were from high income countries. Four studies (McLachlan, Forster, Yelland, Rayner, \& Lumley, 2008; Rayner, Forster, McLachlan, Yelland, \& Davey, 2008; Rayner, McLachlan, Peters, \& Forster, 2013; Schmied, Cooke, Gutwein, Steinlein, \& Homer, 2008) examined, from the perspective of the health professionals, perceptions of the quality of postnatal care provided in hospital. These studies all showed that midwives often experienced dissatisfaction as a result of a lack of adequate staffing or poor skill mix; this in turn created stressful workplace environments which were not conductive to the delivery of quality postnatal care.

Two studies (Beake, Rose, Bick, Weavers, \& Wray, 2010; Fenwick, Butt, Dhaliwal, Hauck, \& Schmied, 2010), both from Australia, examined perceptions of hospital postnatal care from the perspective of women. Both these studies showed that, although women perceived the health professionals positively in the postnatal maternity unit, they identified negative issues such as a lack of staff availability, in particular with regard to breastfeeding support. Health professionals did not necessarily provide accurate information about basic care for the baby at the time of discharge which may well develop the confidence of new parents. These studies revealed that postnatal care in hospital did not focus enough on women's emotional well-being and their long-term health needs and was, in fact, too focused on women with acute medical illness, which was not a typical experience for a majority of women recovering from birth. However, Yelland et al.'s study (2009) from Australia showed that the implementation of strategies to provide more woman-centred care and to improve the continuity of care within the hospital and after the hospital stay through homecare had positive outcomes. The enhancement strategies included: discussions with a midwife during the third trimester of pregnancy to help women plan for the postnatal period, the development of parent written information about the postnatal period, midwife skill-enhancement programmes including communication skills trainings, and creative rostering development allowing midwives to rotate across intrapartum and postnatal care, including hospital-based domiciliary service.

Three studies (Hildingsson \& Sandin-Bojö, 2011; McLachlan et al., 2009; Rayner et al., 2008) revealed that parents may harbour safety concerns especially for the baby as a result of having not enough time to adjust to their parental role during their postnatal stay in hospital. Hildingsson and SandinBojö (2011) found that policies of short postnatal stays after childbirth have often been implemented for economic reasons instead of security or parental reasons, and are a source of dissatisfaction for health professionals as well as for parents (Rayner et al., 2008). The desired length of the hospital stay during the postnatal period depended principally on the parity of the mother. 
According to four studies (Fenwick et al., 2010; Hildingsson \& Sandin-Bojö, 2011; McLachlan et al., 2009; Rayner et al., 2008) first time mothers would expect longer length of stay than other mothers.

Fenwick et al's research, again from Australia, showed that only $50 \%$ of women received home visit support after their inpatient stay, despite a reduced length of postnatal stay for low-risk mothers. Although parents perceived home postnatal midwifery care to be a good option in term of increasing the provision of postnatal care, they were not always willing to trade this for a shorter hospital stay (McLachlan et al., 2009). However, women's satisfaction with their postnatal care was higher when they did, in fact, receive care at home (Fenwick et al. 2010).

\section{What happens within the community?}

Three studies from high-income countries and four studies from low-income countries examined postnatal care model provided within the community.

Fenwick et al.'s study (2010) showed that $80 \%$ of women who received postnatal care at home (community-based) rated it as well/excellent, especially about 'information about breastfeeding', 'supported decision' and 'recognition of ability as a parent and giving confidence skills'. Generally, the women perceived the quality of their care provided at home as being better than in hospital. The community was identified as an appropriate location to provide quality postnatal care that respected traditional cultural practices about the postnatal period (Chien, Tai, Ko, Huang, \& Sheu, 2006). The Gao et al.'s study (2014) also revealed that a Midwifery Group Practice provided in the community provided higher quality, cost effective postnatal care to the Aboriginal mothers who accessed the care than the baseline model of care which occurred essentially in hospital.

There were four studies about low-income areas. In rural areas of India, Paudel et al.'s (2014) showed that three-quarters of women used the existing community postnatal maternity services with a minimum of three visits by a health worker during the postpartum period. Many factors were identified as contributing to postnatal care service utilisation, such as higher level of awareness and education of the mother, higher level of family income, bigger size of family, age for first pregnancy (20-34 years), the use of antenatal care services, and the location of the birth. Probably unsurprisingly, mothers who gave birth at home were less likely to use postnatal care services than those who gave birth within health facility.

Sitrin et al.'s (2013) research in Bangladesh, Malawi and Nepal showed that the rate of postnatal care provided during home visits within the three days after birth decreased from $57 \%$ in Bangladesh to $50 \%$ in Nepal and as low as $10 \%$ in Malawi. Care at home was more likely to be delivered if there was a home visit during the pregnancy and if the birth occurred outside a facility. This model of care that used the existing community services was considered achievable if the health workers were trained, supplied and supervised, and if they were available and connected to the community.

A study by Baqui (2008) in Bangladesh compared the neonatal mortality rates in a population of those receiving postnatal home-care and those receiving community-care (in a community facility) provided by trained health workers in areas with very poor health care access. The study showed that the rate of neonatal mortality in the group receiving home-care decreased by $34 \%$ while the community-care arm had an unchanged neonatal mortality rate. In Bashour et al.'s (2008) study, trained midwives provided home postnatal care at different frequencies after early discharge in Syria (0 visit, 1 visit at day 3 and 4 visits at days 1, 3, 7 and 30). The rate of exclusive breastfeeding up to 
four months of age was higher in the two groups with home-care visits than in the group with no postnatal home-visits.

\section{TYPE OF CARE PROVIDERS - WHO PROVIDES POSTNATAL CARE?}

Different health professionals were identified as postnatal care providers in this analysis. Continuity of carer was an important element of the care provider. For example, Tuominen et al.'s (2012) study showed that parents' satisfaction increased when the health professional providing the postnatal care was the same as the one who provided the antenatal care.

\section{Midwives, nurses and community workers}

Five of the six studies in this theme were in high income settings. Homer et al.'s (2009) study identified the key elements that were required in midwife-led care model according to women and midwives. Midwives were expected to provide safe, supportive and woman-centred care, and to work in collaboration with others when necessary. Midwives considered that the midwife-led model was the ideal model for providing continuity of care. The study by Fenwick et al. (2010) showed that the midwife-led care model provided at home had better or similar patient physical and emotional outcomes, and provided better continuity of care, and more patient-centred care. Three others studies (Gao et al. 2014; Quinn et al. 2013; Tracy et al. 2013) showed that midwife-led care delivered safe and cost-effective care and was suitable for all women; including populations living in rural areas, women who are socially disadvantaged and women with a medical risk. Quinn et al.'s study (2013) showed that local health services were ready to implement midwife-led models in very remote communities; however, Homer et al.'s study (2009) showed that overall the role of the midwife in postnatal care still needed better promotion and greater understanding.

Bashour et al.'s (2008) study is the only low-income country study presenting a midwife-led postnatal care model in this review. Other low-income countries studies used a community workerled model, which may occasionally be conducted by a midwife.

Sitrin et al. (2013) described postnatal care led by community workers in low-income countries. The community workers were family welfare assistants, health surveillance assistants and community health volunteers, who were supposed to live in their catchment area (in $47 \%$ of cases) and who received in-service training on maternal and newborn care. This study also showed that the use of the existing community services was an appropriate solution to improve women and newborns' health outcomes in low-income countries, which suffer from a lack of access to skilled health professionals. However, it also found that health workers needed to be trained and supervised and their connection to the community needed to be carefully maintained. This required improvements in the management of the postnatal home visit at a district level. There were no studies specifically examining a community worker model in high income countries.

\section{Medical model: general practitioners (GPs), obstetricians and specialist obstetricians}

Two studies from high income countries (McLachlan et al., 2008; Quinn et al., 2013) revealed that a high proportion of women used the medical model for their primary maternity care, especially in rural areas where the choice of model of maternity care offered to women was limited. There were no studies addressing this area from low or middle income settings.

Inter-professional collaborative model 
An inter-professional collaborative model was also described as an appropriate way of delivering postnatal care. Munro et al.'s (2013) described primary maternity care providers, being GPs, midwives and nurses, working in collaboration to provide postnatal care in Canadian rural communities. The nurses, who were the local care providers, reported that they considered their skills to be enhanced by the implementation of local midwifery services and by their exposure to the midwives' approach to care. However, the study also revealed the existence of tensions between these groups of health professionals due to factors such as a lack of formal structures for supporting shared care practice, inequities in payment between GPs and midwives, confusion about roles and responsibilities, difference in the scope of practice; and physicians and nurses' negative perceptions of midwifery and homebirth.

Sitrin et al.'s (2013) revealed the need for collaboration between community workers and qualified health professionals to ensure the quality of the postnatal care delivered within the community in a low income setting. Collaboration includes joint management of the workforce and its qualification and the coordination in the provision of postnatal care.

\section{ORGANISATION OF CARE - WHAT IS IMPORTANT IN THE ORGANISATION OF POSTNATAL CARE?}

\section{Patients and professionals' satisfaction}

The satisfaction of women and professionals is a feature that is explicitly examined in high-income countries' studies but not identified in studies from low-income countries. For example, in Sweden, Hildingsson and Sandin-Bojö's (2011) revealed that the two major factors determining a women's satisfaction with postnatal care were the support from staff and the best possible check-up/medical care should this be required. Midwives' perceptions about quality postnatal care principles, as identified in Schmied et al.'s Australian study (2008), centred on the ability to build a relationship with the woman, to individualise the care to her specific needs and to ensure continuity of care. According to the midwives, this would involve an apportioning of 'one-to-one time', which would be described as a daily uninterrupted period of time dedicated exclusively to the woman.

Two major organisational features were identified as being expected during the provision of postnatal care.

\section{Ensuring the continuity of care}

Three studies (Homer et al., 2009; Munro et al., 2013; Tuominen et al., 2012) revealed that women have a strong need for continuity of caregiver. Beake et al.'s study (2010) showed that building the relationship between parents and health professional before the postnatal period improved parents' confidence. Four studies (McLachlan et al., 2008; McLachlan et al., 2009; Tuominen et al., 2012; Yelland et al., 2009) showed that women who had continuity of midwife carer were more satisfied by their postnatal care, as it ensured that their midwives knew them, their story and their needs.

Psaila et al.'s study (2014) revealed that the notion of continuity varied across professional groups: continuity was perceived by midwives and GPs as continuity of carer while nurses promoted a continuity of service model. The study also showed that professionals focused more on continuity within their own service rather than between services. Moreover, midwives, child and family health nurses and GPs believed themselves as being the most appropriate stakeholders to co-ordinate the care. 
In another Australian study, McLachlan et al (2008) found that hospitals attempted to provide some continuity of care in $74 \%$ of cases but found this difficult to achieve. Continuity of carer was encouraged in some hospitals; however they were limited by the models of care that were available in the area, with easy access to community visits with obstetricians and general practitioners and a poor access to midwives. Two studies (Bar-Zeev, Barclay, Farrington, \& Kildea, 2012; Psaila et al., 2014) identified inefficiency of the transmission of information between services during the postnatal period, especially from the birth setting facility to the community health service. Bar-Zeev et al.'s study (2012) revealed that the discontinuities in communication from regional hospital to remote health services was due to the complexity of the discharge process involving multiple stakeholders and task repetition. The study identified a lack of coordination between organisations, a lack of governance and leadership and limited understanding of roles and work practices by rural hospital staff about remote health services. These issues compromised the quality of care and the safety of mothers and newborns, especially for women with vulnerabilities who also encountered an increased risk having discontinuity in care.

Psaila et al.'s study (2014) showed that the transmission of information was identified as particularly important by all health professionals providing postnatal care; however, the opportunity to develop inter-professional relationships was limited, leading to a lack of understanding of the role and the contribution of other involved professional groups. Two studies (Homer et al., 2009; Psaila et al., 2014) showed that collaboration with colleagues was an important aspect of effective transition of care from maternity to child health services. For example, in Psaila et al.'s study (2014), the desire to provide continuity of care led midwives to develop collaborations with child and family nurses during the antenatal period in order for the planning of the postnatal period.

Continuity of care is an important feature in low-income countries as well although less has been written about this setting. However, Sitrin et al.'s study (2013) revealed that building the relationship before birth led to higher rates of utility of postnatal care.

\section{Providing care in a flexible manner to meet women's needs}

The second major feature of postnatal care was the need for flexibility in the provision of care.

Chien et al.'s study (2006) in Taiwan showed that postnatal care provided in respect of cultural rituals was associated with decreased rates of postnatal depression in women. He identified a need for adapting the clinical practice with the cultural background, the beliefs and needs of women in mind. Munro et al's study (2013) showed that the introduction of midwifery practices in rural communities allowed dialogue between stakeholders and, in turn, led to the establishment of standards of care personalised for each community. Three studies (Beake et al. 2010; Rayner et al. 2008; Yelland, Krastev \& Brown 2009) showed that mothers and care providers agreed that postnatal care provided at home was more individualised and flexible than at hospital. Furthermore, four studies (Fenwick et al., 2010; Hildingsson \& Sandin-Bojö, 2011; McLachlan et al., 2009; Rayner et al., 2008) revealed that parents needed more flexibility in the length of stay in postnatal unit at hospital, according to their level of confidence to achieve their parental role after discharge.

A focus on women-centred care was not identified in the four studies of low-income country postnatal care. 


\section{Discussion}

This review has shown that midwives were the predominant providers of postnatal care in all settings. The midwife-led care model was revealed as being safe and cost-effective for postnatal care, as providing positive physical and emotional outcomes, more woman-centred care and better continuity of care than other models of postnatal care. Moreover, this model has been confirmed in the systematic review (Sandall, Soltani, Gates, Shennan, \& Devane, 2013) that identified midwife-led care model as being suitable for all women whatever their social and health status and should be provided to every woman throughout the maternity continuum, including the postnatal period. However, the lack of midwives may lead some countries or settings to resort to using other health professionals or community-health workers. As a result, high-income countries tended to use a medical-led model while low and middle-income countries used a community-services model with a range of workers from various backgrounds or as volunteers. An inter-professional collaborative model was also found to be posed as a solution to workforce shortage, but whilst providing quality postnatal care, this model lacked structural support to facilitate the relationship between providers.

This synthesis has found that the postnatal care occurred in different locations, according to the birth setting, the nature of the woman's choices and the health services available. Hospital was a common setting to provide postnatal care, which was usually provided by midwives. However, overloaded and busy staff, the lack of ability to develop relationships and the limited flexibility of this model made continuity of carer and the provision of woman-centred care aspects difficult. This has, in turn, led to the dissatisfaction for both women and health professionals, and may have resulted in negative impacts on health and safety, especially in the period after discharge. Length of hospital stay tended, therefore, to be reduced, resulting in a reliance on community health workers for providing postnatal care in community health facilities or at home. Home postnatal care was identified as generating similar or better physical and emotional outcomes, higher level of satisfaction for parents and professionals, better continuity of care and carer and more womancentred care. Women giving birth in hospital had better access to postnatal community facility services while women giving birth at home had better access to postnatal home care. However, in overall studies, less than half of women benefited from home postnatal care.

\section{Applicable models of care in low-income countries:}

The ideal postnatal model of care must be provided by skilled professionals to ensure quality of care for both the mother and the baby; and to support, inform and guide parents as they take acquisition of their new role. Midwives are educated and regulated to provide these skills, and many more, and therefore are the most effective ideal provider (Renfrew et al., 2014). Further support and education for midwives is also important. According to the SoWMy (The State of the World's Midwifery, 2014), in many countries, the general public is not aware about the role of the midwife, and the promotion of medical-led model of care as well as the perception that medical-led model provides better social status are real obstacles for the development of midwife-led models of postnatal care. The midwifeled care model should therefore be promoted to both the health professional population and the overall population.

This review has found that the parents' satisfaction is greatest when postnatal care is provided at home by a known health professional. The midwives' qualifications allowed them to practice across 
the full continuum of the maternity period (antenatal, birth and postnatal period) and thus provide continuity of carer. As well, the midwife-led postnatal care model facilitates the creation of a climate of trust between the family and the health practitioner, which helps challenge the perception, some parents may have, that health professionals are not respectful of their cultural and religious beliefs (The State of the World's Midwifery, 2014).

This review has indicated that favouring existing community services is crucial because women already knew and used these services. The improvement in the management of postnatal home visits at a district level has also been identified by Kerber et al.'s review (2007) as an appropriate response to the lack of postnatal care in sub-Saharan, Africa and South Asia. Postnatal care provided in community facilities does not, however, easily reach women who give birth at home. A proportion of women may feel uncomfortable in seeking out this care because of the perception that midwives were too busy or because the community health services may not be accessible in some remote areas. The SoWMY (2014) highlighted the need to invest workers in the community, especially midwives. Midwives are able to work in all settings to reach the most vulnerable mothers and babies (Save the Children, 2013). According to the SoWMY (2014), increasing the recruitment and the deployment of midwives, having effective regulatory bodies and regulatory framework and improving workforce retention of midwives, especially in rural areas, is essential for high quality postnatal care.

The lack of midwives may make midwife-led care model difficult to apply in some areas. A collaborative approach may be an efficient solution to provide quality postnatal care. In low-income countries, resorting to less qualified community health workers instead of midwives or medical professionals is already happening and this needs to be carefully managed and evaluated. A previous review has shown that resorting to task sharing/shifting can improve access to, and the availability of, maternal services in an cost-effectiveness manner however the roles of the different providers needs to be defined (Dawson et al., 2013). To be most efficient and effective, such collaborative health services require improvement in the provision of support and co-ordination between the health professionals in order to ensure appropriate skills mix. It is necessary to focus on the provision of continuing training, supervisory mechanisms and access to equipment and drugs (Dawson et al., 2013). A model that task shifts to the available community health workers with task leading management by a midwife to ensure the coordination, and quality of the care provided may therefore be appropriate to provide postnatal care in area with poor access to maternal health services.

This review showed that while women generally felt that they had benefited from a strong level of support from health professionals during the pregnancy and the birth, they often reported a lack of support during the postnatal period. This period is the determinant for the psychological status of the mother and the time when the foundation links are established between mother and baby and with other family members. There is a need to enhance the attention of postnatal care providers, particularly during their training, about the importance and the content of postnatal care. Midwifery education should thus ensure that postnatal care is given sufficient emphasis and value. In order to promote this, the WHO developed a training handbook 'Counselling for maternal and newborn health care' (2013a), which described the key features of knowledge and practice required for maternity care providers. The handbook focuses on the importance of building appropriate support for the mother and the baby, with the help of the relatives, according to the mother's concerns and 
needs. It also highlights the need to provide women with information in order to enable her to care for the baby and detect danger signs.

Another aspect of the postnatal period that women in our review described as an area needing improvement was the provision of information and care about family planning. As described in the 'essential competencies for basic midwifery practice' (International Confederation of Midwives, 2010), family planning is an important part of the midwives' competencies. The postnatal period is an appropriate period to develop positive parenting and plan future pregnancies, and midwives are the qualified professionals who are able to ensure the provision of culturally sensitive education and high quality contraceptive care, which in turn promotes healthy family life (Homer et al., 2014; The State of the World's Midwifery, 2014). Family planning counselling is an aspect of the public health domain where midwives should have more involvement (Biro, 2011). However, the initiation of effective postpartum contraceptives is an issue that requires greater awareness by health professionals as well as parents, and it is an area where health professionals would benefit from an enhanced knowledge and training (Kestler, Orozco, Palma, \& Flores, 2011; Marlow, Maman, Moodley, \& Curtis, 2013). In order to support this, the WHO handbook for building skills (2013a) has summarised the required health providers' knowledge about family planning and the information to provide to parents.

The lack of research on postnatal care, especially studies from low-income countries, may constitute a limitation toward identifying the most important features of various postnatal models. In the future, research addressing the cultural postnatal and postpartum period in child and maternity is important.

\section{CONCLUSION}

The postnatal period has a determining influence on the health status of both the mother and the baby, and has a significant impact on the overall well-being of the family. Just like the pregnancy and the birth, the postnatal period requires a qualified professional support, although it is often a neglected time in the provision of health quality care. The midwifery-led postnatal care model is an appropriate model of postnatal care for all parts of the world, providing high quality and costeffective care to mother and babies. Midwives are present at all stages of the maternity care continuum and ensure continuity of care and woman-centred care in all settings. Midwife-led postnatal care should therefore be the benchmark model to promote in order to respond to the MDG 4 and 5 and respond to the Actions in the Every Newborn Action Plan (World Health Organization (WHO), 2014). This would require developing the education, practice and regulation of midwives according to the international standards. The lack of qualified midwives and access to postnatal care services may be partially resolved by collaborative models between midwives and the existing health workers and community members. This type of collaboration would involve enhancing the management and support of collaborative teams at a local level. Finally, the provision of postnatal care, together with the family planning counselling, are two components of the maternity period that should be better articulated and expressed during the training and the practice of the relevant health care providers. 


\section{References}

Baqui, A. H., El-Arifeen, S., Darmstadt, G. L., Ahmed, S., Williams, E. K., Seraji, H. R., . . Saha, S. K. (2008). Effect of community-based newborn-care intervention package implemented through two service-delivery strategies in Sylhet district, Bangladesh: a cluster-randomised controlled trial. The Lancet, 371(9628), 1936-1944. doi: 10.1016/S0140-6736(08)60835-1.

Bar-Zeev, S. J., Barclay, L., Farrington, C., \& Kildea, S. (2012). From hospital to home: The quality and safety of a postnatal discharge system used for remote dwelling Aboriginal mothers and infants in the top end of Australia. Midwifery, 28(3), 366-373. doi: http://dx.doi.org/10.1016/j.midw.2011.04.010

Bashour, H. N., Kharouf, M. H., AbdulSalam, A. A., El Asmar, K., Tabbaa, M. A., \& Cheikha, S. A. (2008). Effect of postnatal home visits on maternal/infant outcomes in Syria: a randomized controlled trial. Public Health Nursing, 25(2), 115-125. DOI: 10.1111/j.15251446.2008.00688.x.

Beake, S., Rose, V., Bick, D., Weavers, A., \& Wray, J. (2010). A qualitative study of the experiences and expectations of women receiving in-patient postnatal care in one English maternity unit. BMC Pregnancy and Childbirth, 10(1), 70. doi: 10.1186/1471-2393-10-70.

Biro, M. A. (2011). What has public health got to do with midwifery? Midwives' role in securing better health outcomes for mothers and babies. Women and Birth, 24(1), 17-23. doi: 10.1016/j.wombi.2010.06.001

CASP UK. (2014). Critical Appraisal Skills Programme - Making sense of evidence. Retrieved 14/08/2014, from http://www.casp-uk.net/\#!casp-tools-checklists/c18f8

Chien, L. Y., Tai, C. J., Ko, Y. L., Huang, C. H., \& Sheu, S. J. (2006). Adherence to “Doing-the-month" practices is associated with fewer physical and depressive symptoms among postpartum women in Taiwan. Research in Nursing \& Health, 29(5), 374-383. doi: 10.1002/nur.20154.

Dawson, A. J., Buchan, J., Duffield, C., Homer, C. S., \& Wijewardena, K. (2013). Task shifting and sharing in maternal and reproductive health in low-income countries: a narrative synthesis of current evidence. Health Policy and Planning, czt026. doi: 10.1016/j.ijgo.2013.02.007.

Fenwick, J., Butt, J., Dhaliwal, S., Hauck, Y., \& Schmied, V. (2010). Western Australian women's perceptions of the style and quality of midwifery postnatal care in hospital and at home. Women and Birth, 23(1), 10-21. doi: 10.1016/j.wombi.2009.06.001.

Gao, Y., Gold, L., Josif, C., Bar-Zeev, S., Steenkamp, M., Barclay, L., . . Kildea, S. (2014). A costconsequences analysis of a Midwifery Group Practice for Aboriginal mothers and infants in the Top End of the Northern Territory, Australia. Midwifery, 30(4), 447-455. doi: 10.1016/j.midw.2013.04.004.

Hildingsson, I. M., \& Sandin-Bojö, A.-K. (2011). 'What is could indeed be better'-Swedish women's perceptions of early postnatal care. Midwifery, 27(5), 737-744. doi: 10.1016/j.midw.2010.04.007.

Homer, C. S., Friberg, I. K., Dias, M. A. B., ten Hoope-Bender, P., Sandall, J., Speciale, A. M., \& Bartlett, L. A. (2014). The projected effect of scaling up midwifery. The Lancet. doi: 10.1016/S0140-6736(14)60790-X. 
Homer, C. S., Passant, L., Brodie, P. M., Kildea, S., Leap, N., Pincombe, J., \& Thorogood, C. (2009). The role of the midwife in Australia: views of women and midwives. Midwifery, 25(6), 673-681. DOI: 10.1016/j.midw.2009.06.014.

International Confederation of Midwives. (2010). Essential competencies for basic midwifery practice. The Hague, The Netherlands: ICM. doi: 10.1016/j.midw.2011.03.005.

Kerber, K. J., de Graft-Johnson, J. E., Bhutta, Z. A., Okong, P., Starrs, A., \& Lawn, J. E. (2007). Continuum of care for maternal, newborn, and child health: from slogan to service delivery. The Lancet, 370(9595), 1358-1369. doi: 10.1016/S0140-6736(07)61578-5.

Kestler, E., Orozco, M. d. R., Palma, S., \& Flores, R. (2011). Initiation of effective postpartum contraceptive use in public hospitals in Guatemala. Revista Panamericana de Salud Pública, 29(2), 103-107.

Marlow, H. M., Maman, S., Moodley, D., \& Curtis, S. (2013). Postpartum Family Planning Service Provision in Durban, South Africa: Client and Provider Perspectives. Health Care for Women International, 35(2), 175-199. doi: 10.1080/07399332.2013.815753.

McLachlan, H. L., Forster, D. A., Yelland, J., Rayner, J., \& Lumley, J. (2008). Is the organisation and structure of hospital postnatal care a barrier to quality care? Findings from a state-wide review in Victoria, Australia. Midwifery, 24(3), 358-370. doi: 10.1016/j.midw.2006.10.006

McLachlan, H. L., Gold, L., Forster, D. A., Yelland, J., Rayner, J., \& Rayner, S. (2009). Women's views of postnatal care in the context of the increasing pressure on postnatal beds in Australia. Women and Birth, 22(4), 128-133. doi: 10.1016/j.wombi.2009.04.003.

Munro, S., Kornelsen, J., \& Grzybowski, S. (2013). Models of maternity care in rural environments: Barriers and attributes of interprofessional collaboration with midwives. Midwifery, 29(6), 646-652. doi: 10.1016/j.midw.2012.06.004.

Paudel, D. P., Nilgar, B., \& Bhandankar, M. (2014). Determinants of postnatal maternity care service utilization in rural Belgaum of Karnataka, India: A community based cross-sectional study. International Journal of Medicine \& Public Health, 4(1). doi: 10.4103/2230-8598.127167.

Psaila, K., Schmied, V., Fowler, C., \& Kruske, S. (2014). Discontinuities between maternity and child and family health services: health professional's perceptions. BMC Health Services Research, 14(1), 4. doi:10.1186/1472-6963-14-4.

Quinn, E., Noble, J., Seale, H., \& Ward, J. E. (2013). Investigating the potential for evidence-based midwifery-led services in very remote Australia: Viewpoints from local stakeholders. Women and Birth, 26(4), 254-259. doi: 10.1016/j.wombi.2013.07.005.

Rayner, J.-A., Forster, D., McLachlan, H., Yelland, J., \& Davey, M.-A. (2008). A state-wide review of hospital postnatal care in Victoria, Australia: the views and experiences of midwives. Midwifery, 24(3), 310-320. doi: 10.1016/j.midw.2006.10.008.

Rayner, J.-A., McLachlan, H. L., Peters, L., \& Forster, D. A. (2013). Care providers' views and experiences of postnatal care in private hospitals in Victoria, Australia. Midwifery, 29(6), 622-627. doi: 10.1016/j.midw.2006.10.008. 
Renfrew, M. J., McFadden, A., Bastos, M. H., Campbell, J., Channon, A. A., Cheung, N. F., . . Malata, A. (2014). Midwifery and quality care: findings from a new evidence-informed framework for maternal and newborn care. The Lancet. doi: 10.1016/S0140-6736(14)60789-3.

Sandall, J., Soltani, H., Gates, S., Shennan, A., \& Devane, D. (2013). Midwife-led continuity models versus other models of care for childbearing women. Cochrane Database of Systematic Reviews(8). doi: 10.1080/02646830701494474.

Save the Children. (2013). Surviving the first day - state of the world's mother 2013. London: Save the Children.

Schmied, V., Cooke, M., Gutwein, R., Steinlein, E., \& Homer, C. (2008). Time to listen: strategies to improve hospital-based postnatal care. Women and Birth, 21(3), 99-105. doi: 10.1016/j.wombi.2008.04.002.

Shahheidari, M., \& Homer, C. (2012). Impact of the design of neonatal intensive care units on neonates, staff, and families: a systematic literature review. The Journal of Perinatal \& Neonatal Nursing, 26(3), 260-266. doi: 10.1097/JPN.0b013e318261ca1d.

Sitrin, D., Guenther, T., Murray, J., Pilgrim, N., Rubayet, S., Ligowe, R., . . Moran, A. (2013). Reaching Mothers and Babies with Early Postnatal Home Visits: The Implementation Realities of Achieving High Coverage in Large-Scale Programs. PLOS ONE, 8(7), doi: 10.1371/journal.pone.0068930.

The State of the World's Midwifery. (2014). A universal pathway. A women's right to health. USA: United Nations Population Fund.

Tuominen, M., Kaljonen, A., Ahonen, P., \& Rautava, P. (2012). Does the organizational model of the maternity health clinic have an influence on women's and their partners' experiences? A service evaluation survey in Southwest Finland. BMC Pregnancy and Childbirth, 12(1), 96. doi: 10.1186/1471-2393-12-96.

World Health Organization. (2013a). Counselling for maternal and newborn health care: a handbook for building skills. Geneva: WHO.

World Health Organization. (2013b). WHO recommendations on postnatal care of the mother and newborn. Geneva: WHO.

World Health Organization. (2014). 10 facts on maternal health. Retrieved 06 July 2014, from http://www.who.int/features/factfiles/maternal_health/en/

World Health Organization (WHO). (2014). Every newborn: an action plan to end preventable deaths. Geneva: WHO.

Yelland, J., Krastev, A., \& Brown, S. (2009). Enhancing early postnatal care: findings from a major reform of maternity care in three Australian hospitals. Midwifery, 25(4), 392-402. doi: 10.1016/j.midw.2007.01.004. 